\title{
Interactive Genetic Algorithms with Grey Level of Individual Interval
}

\section{Fitness}

\author{
Guo Guang-song, Liu Yuan-peng \\ School of Mechatronics Engineering \\ Zheng Zhou Institute of Aeronautical Industry Management China \\ Guogs78@126.com
}

Key words:interval; fitness; grey level; interaction genetic algorithms

\begin{abstract}
For the problem that interactive genetic algorithms lack a way of measuring uncertainty of comment, a method with grey level for uncertainty of individuals evolutionary is proposed in this paper in which the individual fitness is an interval. Through analyzing these fitness grey level, information reflecting the distribution of an evolutionary population is abstracted. Based on these, the adaptive probabilities of crossover and mutation operation of an evolutionary individual are proposed.
\end{abstract}

\section{INTRODUCTION}

Interactive genetic algorithms (IGAs), proposed in middle 1980s, are effective methods for solving optimization problems with implicit indices. Generally speaking, there are 2 kinds of evaluation methods on evolutionary individuals as follows. One is that human evaluates evolutionary based on individuals fitness estimates and the other is extraction of cognitive information to guide the evolution of operating. For the former, Biles adopted neural networks to learn human's intelligent evaluation on evolutionary individuals, and the number of evolutionary individuals evaluated by human is reduced by use of neural networks rather than human evaluating evolutionary individuals in appropriate time [1]. Hao et al. did it based on "the fitness" of gene sense units [2]. For the latter idea, [3]presented the interval fitness evaluation, which using interval dominance select individuals who strongly reflects the ambiguity and progressive of human cognitive. Although these studies improve the algorithm performance, significantly reducing the people's fatigue, but they don't give the explicit quantitative judgments of the uncertainty, and uncertainty is important for individual evaluation in interactive genetic algorithm, so exploring the uncertainty in interactive genetic algorithm is an important issue.

\section{GREY NUMBER AND GREY LEVEL}

The grey number and grey level are an important part of uncertainty analysis, it is the mathematical basis for this article. Therefore, this section gives the definition of grey number and grey level.

Definition 1[4]: Let $\wp(\theta)$ be a information range of $\wp, D$ be a numerical range, $\otimes$ be an uncertainty number of $\wp$, let $d^{o}$ be a latent truth-value of $\otimes, L^{*}$ be guide rule for criticize truth-value $d^{*}$,let $\tilde{\otimes}$ be a measured number for $\wp$,if

$$
\forall \tilde{\otimes} \in \otimes \Rightarrow \tilde{\otimes} \in \wp, \wp=\left\{\tilde{\otimes} \mid \exists d^{\circ}, \tilde{\otimes} \operatorname{Apr} \wp(\theta), d^{\circ} \operatorname{Apr} \wp(\theta), \tilde{\otimes} \in \tilde{D} \subset D\right\}
$$

where $\mathrm{Apr}$ represents implied subfield, then, $\otimes$ is a grey number of $\wp ; \tilde{\otimes}$ is a whitening number of $\otimes ; \tilde{D}$ is numerical coverage of $\otimes ; \wp(\theta)$ and $L^{*}$ are information background of $\otimes$, when $\tilde{D}$ is a continuous set, $\otimes$ is said to be continuous grey number.

Definition 2[4]: If $\otimes$ is continuous measured number, $\tilde{D}$ is numerical coverage of $\otimes, P O T_{\sim}$ is continuous spectral mapping, $h_{\sim r}^{o}$ is said to be grey level of ${ }^{\otimes}$,provided that

$$
h_{\sim r}^{o}=g_{\sim D^{\prime}}^{o}, \quad g_{\sim D^{\prime}}^{o}=P O T_{\sim} D^{\prime}, \quad D^{\prime}=[\alpha, 1]=F(\tilde{D}),
$$

$F$ is said to be conversion from $\tilde{D}$ to $D^{\prime}$. 


\section{Methodolgy OF The Algorithms}

\section{A. Individual interval fitness [3]}

Let the $i$ th individual of a population in the $t$ th generation be $x_{i}(t), i=1,2, \cdots, N$, and the population size be N. Because of fuzzy human's cognition on $x_{i}(t)$, one can hardly assign $x_{i}(t)$ 's fitness exactly,but does assign its range easily,which can be expressed with an interval. Therefore, $x_{i}(t)$ 's fitness can be described as follows.

$$
f\left(x_{i}(t)\right)=\left[\underline{f}\left(x_{i}(t)\right), \bar{f}\left(x_{i}(t)\right)\right]
$$

Where $\underline{f}\left(x_{i}(t)\right), \bar{f}\left(x_{i}(t)\right)$ are the lower limit and the upper limit of human's evaluation on $x_{i}(t)$, respectively.

\section{B. Grey level of interval fitness}

Because of fuzzy human's cognition, it is difficult to determine the fitness value of $x_{i}(t)$ accurately, so $f\left(x_{i}(t)\right)$ is an uncertain number. It is obvious that human cognition is the information background in the process of human-computer interaction. In the evaluation criterion of preference, according to definition 2 in section 2, the true-value $f^{*}\left(x_{i}(t)\right.$ ) (satisfactory solution) of individual $x_{i}(t)$ can be considered measured grey level. When described the individual fitness with interval number, the $\underline{f}\left(x_{i}(t)\right)$ and $\bar{f}\left(x_{i}(t)\right)$ become preference data which reflect of the cognition. However, because the cognitive information is not complete, $\underline{f}\left(x_{i}(t)\right)$ and $\bar{f}\left(x_{i}(t)\right)$ are only implied solution to preference, so $\underline{f}\left(x_{i}(t)\right)$ and $\bar{f}\left(x_{i}(t)\right)$ constitute the whitening number of $f^{*}\left(x_{i}(t)\right)$. A bounded continuous interval $f\left(x_{i}(t)\right)=\left[\underline{f}\left(x_{i}(t)\right), \bar{f}\left(x_{i}(t)\right)\right]$ become numerical coverage of $f^{*}\left(x_{i}(t)\right)$, and $f^{*}\left(x_{i}(t)\right)$ is a continuous measured grey number. As $f\left(x_{i}(t)\right)$ is the set of all whitening number of $f^{*}\left(x_{i}(t)\right)$, there must be a value which reflects the true-value that people most satisfied with the evaluation in interval $f\left(x_{i}(t)\right)$. Then the actual fitness $f^{*}\left(x_{i}(t)\right)$ of $x_{i}(t)$ must be in interval $\left[\underline{f}\left(x_{i}(t)\right), \bar{f}\left(x_{i}(t)\right)\right]$.If let grey level of $f^{*}\left(x_{i}(t)\right)$ is $h\left(x_{i}(t)\right)$, as $f\left(x_{i}(t)\right)$ is a bounded interval, according to the definition 2 in section 2 ,then one has

$$
h\left(x_{i}(t)\right)=1-\frac{f\left(x_{i}(t)\right)}{\bar{f}\left(x_{i}(t)\right)}
$$

The grey level of current generation of the population evolution is $h(t)$

$$
h(t)=\frac{1}{N} \sum_{i=1}^{N} h\left(x_{i}(t)\right)
$$

Where $h(t)$ reflects the uncertainty of human evaluation. In general, human preferences is different with different individuals, the fitness value given is not the same. Easy to see, in the early evolution, human cognition is rather ambiguous on $x_{i}(t)$, the uncertainty of evolution is larger, $w\left(f\left(x_{i}(t)\right)\right)$ is large too. The whitning number is more among $f\left(x_{i}(t)\right)$, then $h\left(x_{i}(t)\right)$ is larger. As the population continues to evolve, cognition will become clearer and clearer, hence the evaluation of uncertainty became smaller, the whitening number will be fewer in $f\left(x_{i}(t)\right)$, then $h\left(x_{i}(t)\right)$ gradually become smaller, $w\left(f\left(x_{i}(t)\right)\right)$ is more and more narrow. When human evaluation is a single numerical assessment, the fitness is still an uncertain number affected by noise, while the whitening number of $f^{*}\left(x_{i}(t)\right)$ is only, then the individual fitness can be considered a discrete measured grey number, and grey level is 0 .

\section{Probabilites of crossover and mutation operators}

- Probability of crossover operator

The ideas of the probability of crossover operator proposed in this paper is as follows: (1) if $h\left(x_{i}(t)\right)$ and $h(t)$ are large, it means that the uncertainty of individual fitness is great, then increase the crossover probability, otherwise, decrease it . (2) In the late of evolution, with the evolution of generation increases, the gap of $h\left(x_{i}(t)\right)$ and $h(t)$ decrease gradually. Thus, the crossover probability should be reduced to ensure convergence. Based on the above ideas, the design of the crossover probability is as follows:

$$
p_{c}\left(x_{i}^{\prime}(t)\right)=\frac{1}{1+\exp \left(-k_{1} \cdot \frac{T}{t} \cdot \frac{h\left(x_{i}(t)\right)}{h(t)}\right)}
$$


Where $T$ is the number of termination generation, $k_{1}$ is an adjustment coefficient

Considering the parents $x_{i}^{\prime}(t)$ and $x_{j}^{\prime}(t)$, calculate the mutation probability $p_{m}\left(x_{i}^{\prime}(t)\right)$ and $p_{\mathrm{c}}\left(x_{j}^{\prime}(t)\right)$ according to Formula (5), whichever the greater of the two bodies as a crossover probability ,then perform mutation operation.

\section{- Probability of mutation operator}

The idea of the probability of mutation operator presented in this paper is as follows: (1) If $h(t)$ and $h\left(x_{i}(t)\right)$ are small,it means that the uncertainty of individual fitness is great,then increase the mutation probability, otherwise, decrease it. (2) In the late of evolution, the purpose of evolutionary is to ensure convergence. Thus, with the evolution of generation increases, the mutation probability should be reduced. For this reason, mutation probability will be limited in interval $(0,0.5)$. Based on the above ideas, the design of the mutation probability is as follows:

$$
p_{m}\left(x_{i}^{\prime}(t)\right)=1-\frac{1}{1+\exp \left(-k_{2} \cdot \frac{T}{t} \cdot \frac{h(t)}{h\left(x_{i}(t)\right)}\right)}
$$

Where $k_{2}$ is an adjustment coefficient.

Considering the parents $x_{i}^{\prime}(t)$, calculate the mutation probability $p_{m}\left(x_{i}^{\prime}(t)\right)$ according to Formula (5), then perform mutation operation.

- Steps of the algorithm

In this paper, Interactive Genetic Algorithms with Grey Level of Individual Interval Fitness(IGAs-GLIIF) was proposed .Its steps are as follows:

Step1 Set the parameters in the algorithm. Let $t=0$, and initialize an evolutionary population;

Step2 Decode and assign the interval fitness of individuals based on human evaluation;

Step3 Calculate the gray level of interval fitness according to formula (2) and (3);

Step4 Produce parents by tournament selection;

Step5 Perform crossover and mutation operators according to Formula (4) and (5), and produce off-spring $x(t)$. Let $\mathrm{t}=\mathrm{t}+1$;

Step6 Judge if the termination criterion is met, if yes, then go to step 7; otherwise go to step 2;

Step7 Output the optima and stop the algorithm.

\section{APPLICATIONS IN A FASHION EVOLUTIONARY DESIGN SYSTEM}

\section{A. Backgrounds}

The goal of fashion design is to find "the most satisfactory fashion". However different people have different opinions on it, therefore it is impossible to get a uniform and explicit index, so it is infeasible for traditional GAs to deal with fashion design, but it is suitable for IGAs to do.

Each individual in the system is a style of fashion composed of coat and skirt, and is encoded with a binary string of 18 bits, where the first 5 bits expresses the style of coat, the 6th to 10th bits expresses the style of skirt, the 11th to 14th bits expresses the color of coat, and the last 4 bits expresses the color of skirt. There are 32 kinds of styles for coat and skirt, and their names correspond to the integersfrom 0 to 31 , which are also their decimals of these binarycodes.According to a human's preference, the system will look for"the most satisfactory fashion" in the search space with $2^{5} \times 2^{5} \times 2^{4} \times 2^{4}=262,144$ individuals in evolutionary optimization.

\section{B. $\quad$ Parameters Settings}

Algorithms for comparison is the traditional interactive genetic algorithm (IGAs), compared algorithms' performance indicators including the number of evolution generation and satisfactory solution. The probabilities of crossover and mutation of IGAs are shown as Table 1 . In Formula (5) and (6) $, k_{1}=k_{2}=9, T=20$.Besides, the population size is set to 8, and the scope of the lower limit and the upper limit of interval fitness is $0-100$. When the evolution converges or human is satisfied with the optimal results, the evolution can be stopped manually. 


\section{Performance Analysis}

First of all, considering this proposed algorithm, when the evolution terminates, statistics of all this running to find the number of satisfactory solution are done, with the algorithm 20 times independently, statistical results are listed in Table 2.

Then consider the IGAs, aim to different crossover and mutation probability in Table 1 , the algorithm runs 20 times independently,statistics of the average number of evolution generation and satisfactory solutions are listed in Table 3.

As can be seen from Table 3, when the crossover and mutation operator increases, the convergence rate of IGAs will slow down, thus the evolving generation increase; when the crossover operator is constant while the mutation operator increases, the number of evolution generation and satisfactory solution of IGAs increase; when the mutation operator unchanged while the crossover operator increase, the number of evolution generation and satisfactory solution of IGAs also add, but the latter has greater impact in the number of evolution generation and satisfaction solution.

TABLE 1 SETTING OF PARAMETERS

\begin{tabular}{llllllllll}
\hline & 1 & 2 & 3 & 4 & 5 & 6 & 7 & 8 & 9 \\
\hline$p_{\mathrm{c}}$ & 0.6 & 0.6 & 0.6 & 0.7 & 0.7 & 0.7 & 0.8 & 0.8 & 0.8 \\
$p_{\mathrm{m}}$ & 0.02 & 0.04 & 0.1 & 0.02 & 0.04 & 0.1 & 0.02 & 0.04 & 0.1 \\
\hline
\end{tabular}

TABLE 2 NO.OF EVOLUTION GENERATION AND SATISFACTORY SOLUTIONS OF IGAS-GLIIF PER GENERATION

\begin{tabular}{lll}
\hline $\begin{array}{c}\text { The amount of } \\
\text { experiments }\end{array}$ & $\begin{array}{c}\text { Evolution } \\
\text { generation }\end{array}$ & $\begin{array}{c}\text { Amount of selected local preference } \\
\text { individual }\end{array}$ \\
\hline 1 & 12 & 42 \\
2 & 10 & 39 \\
3 & 10 & 37 \\
4 & 11 & 41 \\
5 & 13 & 44 \\
6 & 10 & 38 \\
7 & 13 & 46 \\
8 & 11 & 38 \\
9 & 12 & 43 \\
10 & 11 & 39 \\
11 & 9 & 36 \\
12 & 12 & 43 \\
13 & 12 & 42 \\
14 & 8 & 34 \\
15 & 10 & 37 \\
16 & 9 & 38 \\
17 & 11 & 42 \\
18 & 11 & 44 \\
19 & 10 & 41 \\
20 & 14 & 46 \\
Average & 10.95 & 40.5 \\
\hline
\end{tabular}

TABLE 3 NO.OF EVOLUTION GENERATION AND SATISFACTORY SOLUTIONS OF IGAS

\begin{tabular}{|c|c|c|c|c|c|c|c|c|c|}
\hline Indicator & 1 & 2 & 3 & 4 & 5 & 6 & 7 & 8 & 9 \\
\hline $\begin{array}{c}\text { The average amount } \\
\text { of evolution } \\
\text { generation }\end{array}$ & 9.75 & 9.88 & 10.21 & 10.37 & 10.42 & 10.44 & 10.61 & 11.2 & 11.32 \\
\hline $\begin{array}{c}\text { The average amount } \\
\text { of satisfactory } \\
\text { solutions }\end{array}$ & 23.54 & 24.8 & 26.57 & 26.72 & 27.49 & 29.62 & 30.22 & 32.17 & 33.06 \\
\hline
\end{tabular}




\section{Conclution}

Compared to the traditional interactive genetic algorithm, this paper proposed the adaptive interactive genetic algorithm based on fitness grey level. Its characteristics are mainly embodied in the following two aspects: one is that a grey level is adopted to measure the uncertainty of evolution, reflecting the process of human cognitive; the other is that a grey level is adopted to make crossover and mutation probability change adaptively according to population evolution, maintaining the diversity of the population evolutionary, offering a basis to find more satisfactory solution.

\section{REFERENCES}

[1] Biles J. A, Anderson P. G and Loggi L.W, "Neural network fitness functions for a musical IGA," Proceedings of the International Symposium on Intelligent Industrial Automation and Soft Computing, 1996,pp. 39-44.

[2] Guo-sheng HAO, Dun-wei GONG and You-qun SHI, "Search Space Partition Based on Autonomous Genetic Algorithm and Its Application," Journal of Hangzhou Institute of Electronic Engineering, 2005,25(5),pp.6-9.

[3] Dunwei Gong, Guangsong Guo, “Adaptive Interactive Genetic Algorithms with Interval Fitness of Evolutionary Individuals,’Progress in Natural Science ,2008,18(3),pp. 359-365.

[4] Ju-long Deng, Element of Grey Theory. Wuhan: HUST Press, 2003. 